\title{
A characterization of graphs by codes from their incidence matrices
}

\author{
Peter Dankelmann \\ Department of Mathematics \\ University of Johannesburg \\ P.O. Box 524 \\ Auckland Park 2006, South Africa \\ pdankelmann@uj.ac.za \\ Jennifer D. Key Bernardo G. Rodrigues \\ School of Mathematical Sciences \\ University of KwaZulu-Natal \\ Durban 4041, South Africa \\ keyj@clemson.edu \\ rodrigues@ukzn.ac.za
}

Submitted: Oct 3, 2012; Accepted: Aug 4, 2013; Published: Aug 16, 2013

Mathematics Subject Classifications: 05B05, 94B05

\begin{abstract}
We continue our earlier investigation of properties of linear codes generated by the rows of incidence matrices of $k$-regular connected graphs on $n$ vertices. The notion of edge connectivity is used to show that, for a wide range of such graphs, the $p$-ary code, for all primes $p$, from an $n \times \frac{1}{2} n k$ incidence matrix has dimension $n$ or $n-1$, minimum weight $k$, the minimum words are the scalar multiples of the rows, there is a gap in the weight enumerator between $k$ and $2 k-2$, and the words of weight $2 k-2$ are the scalar multiples of the differences of intersecting rows of the matrix. For such graphs, the graph can thus be retrieved from the code.
\end{abstract}

Keywords: codes; graphs; edge-connectivity

\section{Introduction}

Linear codes generated by $|V| \times|E|$ incidence matrices for several classes of regular graphs $\Gamma=(V, E)$ were examined in, for example, $[13,22,20,21,15]$, and shown to have certain important common properties. In order to establish this common property more generally, in [8] it was shown that several properties of these codes for regular connected graphs can 
be derived from properties of the graph involving edge cuts, i.e. the removal of a set $S$ of edges of the graph $\Gamma$ and the ensuing properties of the graph $\Gamma-S$ that excludes these edges. For a prime $p$, denote by $C_{p}(G)$ the $p$-ary code generated by the rows of a $|V| \times|E|$ incidence matrix $G$ of a regular connected graph $\Gamma$. It was shown in [8] that if $\Gamma$ satisfies any of an extensive list of conditions, the minimum distance of $C_{2}(G)$ equals the edge-connectivity $\lambda(\Gamma)$ of $\Gamma$, i.e., the smallest number of edges whose removal renders $\Gamma$ disconnected, and there are no words whose weight is strictly between $\lambda(\Gamma)$ and $\lambda^{\prime}(\Gamma)$, where the restricted edge-connectivity $\lambda^{\prime}(\Gamma)$ is defined as the smallest number of edges whose removal results in a graph that is disconnected, and in which every component has at least two vertices. The analogous results for $p$-ary codes, for $p$ an odd prime, in that paper included a much smaller class of graphs, even though it had been shown, by different methods (in $[13,22,20,21,15]$, for example) that the same results hold for all primes for all the classes studied. Thus we continue our study here to broaden our results for the $p$-ary case where $p$ is odd. The dimension of $C_{p}(G)$ is well-known to be $|V|$ or $|V|-1$ : see Result 1 .

In addition it has also been observed for several classes of connected $k$-regular graphs that the words of weight $2 k-2$ in $C_{p}(G)$, for any prime $p$, are the scalar multiples of the difference of two intersecting rows of the incidence matrix $G$. Using edge cuts we are able to prove this generally for a wide class of graphs. These properties of the codes (the minimum weight, the nature of the minimum words, the gap in the weight enumerator, the nature of the words of the second smallest weight) from an incidence matrix of a graph are reminiscent of the properties of the codes from incidence matrices of desarguesian projective planes, except that in that case only a prime dividing the order of the plane will produce such properties: see, for example, [1, Chapter 5]. We note also that although the minimum weight and the nature of the minimum words for the $p$-ary codes from arbitrary finite projective planes of order divisible by $p$ have this property, the existence of the gap and the nature of the words of weight the same as that from the difference of two rows of an incidence matrix, is not necessarily achieved for non-desarguesian planes: see [14] for some counter-examples.

Our main results for the codes are then Theorem 11 (in Section 4) for the binary codes, Theorem 16 (in Section 6) for the bipartite $p$-ary case, $p$ odd, and Corollaries 30, 31 (in Section 6 ) for the non-bipartite $p$-ary case, $p$ odd. These results are established in the sections that follow. Background definitions and previous results are given in Section 2. In Section 7 we give a short summary of what we have broadly achieved.

\section{Background and notation}

\subsection{Codes from designs}

For notation on designs and codes we refer to [1]. An incidence structure $\mathcal{D}=(\mathcal{P}, \mathcal{B}, \mathcal{J})$, with point set $\mathcal{P}$, block set $\mathcal{B}$ and incidence $\mathcal{J}$ is a $t$ - $(v, k, \lambda)$ design, if $|\mathcal{P}|=v$, every block $B \in \mathcal{B}$ is incident with precisely $k$ points, and every $t$ distinct points are together incident with precisely $\lambda$ blocks. The code $C_{F}(\mathcal{D})$ of the design $\mathcal{D}$ over the finite field $F$ 
is the space spanned by the incidence vectors of the blocks over $F$. If $F=\mathbb{F}_{p}$ then we write $C_{p}(\mathcal{D})$ for $C_{F}(\mathcal{D})$.

All the codes here are linear codes, and the notation $[n, k, d]_{q}$ will be used for a code $C$ over $\mathbb{F}_{q}$ of length $n$, dimension $k$, and minimum weight $d$, where the weight wt $(v)$ of a vector $v$ is the number of non-zero coordinate entries. The support, $\operatorname{Supp}(v)$, of a vector $v$ is the set of coordinate positions where the entry in $v$ is non-zero. So $|\operatorname{Supp}(v)|=\operatorname{wt}(v)$. The vector all of whose entries are 1 is the all-one vector, denoted by $\boldsymbol{\jmath}$. A generator matrix for a code $C$ is a $k \times n$ matrix made up of a basis for $C$. We call two codes isomorphic if they can be obtained from one another by permuting the coordinate positions.

\subsection{Graphs and codes}

The graphs, $\Gamma=(V, E)$ with vertex set $V$, or $V(\Gamma)$, and edge set $E$, or $E(\Gamma)$, discussed here are undirected with no loops and no multiple edges. The order of $\Gamma$ is $|V|$. If $x, y \in V$ and $x$ and $y$ are adjacent, we write $x \sim y$, and $x y$ for the edge in $E$ that they define. The set of neighbours of $u \in V$ is denoted by $N(u)$, and the valency or degree of $u$ is $|N(u)|$, which we denote by $\operatorname{deg}_{\Gamma}(u)$. The minimum and maximum degrees of the vertices of $\Gamma$ are denoted by $\delta(\Gamma)$ and $\Delta(\Gamma)$, respectively. The graph is $k$-regular, where $k \in \mathbb{N}_{0}$, if all its vertices have degree $k$, and $\Gamma$ is said to be regular if it is $k$-regular for some $k$. If $S \subseteq E$ then $\Gamma-S=(V, E-S)$, and $\Gamma[S]$ is the subgraph induced by $S$, i.e. the subgraph of $\Gamma$ whose vertex set consists of the vertices of $\Gamma$ that are incident with an edge in $S$, and whose edge set is $S$.

If for some $r \geqslant 2, x_{i} x_{i+1}$ for $i=1$ to $r-1$, are all edges of $\Gamma$, and the $x_{i}$ are all distinct, then the sequence written $\left(x_{1}, \ldots, x_{r}\right)$ will be called a path in $\Gamma$. If also $x_{r} x_{1}$ is an edge, then this is a closed path, circuit or cycle, of length $r$, also written $C_{r}$. If for every pair of vertices there is a path connecting them, the graph is connected. A perfect matching is a set $S$ of disjoint edges such that every vertex is on exactly one member of $S$. The girth $g(\Gamma)$ of $\Gamma$ is the length of a shortest cycle in $\Gamma$. The distance $d(u, v)$ between two vertices $u$ and $v$ of a graph $\Gamma$ is the minimum length of a path from $u$ to $v$. The diameter of $\Gamma$, denoted by $\operatorname{diam}(\Gamma)$ is the largest of all distances between vertices of $\Gamma$, i.e., $\operatorname{diam}(\Gamma)=\max _{u, v \in V(\Gamma)} d(u, v)$. A subgraph $\Gamma^{\prime}$ of a graph $\Gamma$ is isometric if for any two vertices of $\Gamma^{\prime}$ their distance apart in $\Gamma^{\prime}$ is the same as that in $\Gamma$.

A strongly regular graph $\Gamma$ of type $(n, k, \lambda, \mu)$ is a regular graph on $n=|V|$ vertices, with valency $k$ which is such that any two adjacent vertices are together adjacent to $\lambda$ vertices and any two non-adjacent vertices are together adjacent to $\mu$ vertices. If $\Gamma$ is strongly regular of type $(n, k, \lambda, \mu)$ then the complement $\Gamma^{c}$ is strongly regular of type $(n, n-k-1, n-2 k+\mu-2, n-2 k+\lambda)$. The parameters for $\Gamma$ are linked by the equation

$$
(n-k-1) \mu=k(k-\lambda-1) \text {. }
$$

To avoid trivial cases, we require that a strongly regular graph and its complement are both connected, and so $0<\mu<k<n-1$. Furthermore, we exclude the complete and the null graphs. 
An adjacency matrix $A=\left[a_{u, v}\right]$ of a graph $\Gamma=(V, E)$ is a $|V| \times|V|$ matrix with entries $a_{u, v}, u, v \in V$, such that $a_{u, v}=1$ if $u \sim v$ and $a_{u, v}=0$ otherwise. An incidence matrix of $\Gamma$ is an $|V| \times|E|$ matrix $G=\left[g_{u, e}\right]$ with $g_{u, e}=1$ if the vertex $u$ is on the edge $e$ and $g_{u, e}=0$ otherwise. We denote the row of $G$ corresponding to vertex $v$ by $G_{v}$.

If $\Gamma$ is regular with valency $k$, then the $1-(|E|, k, 2)$ design with incidence matrix $G$ is called the incidence design of $\Gamma$. It was proved in [13] that if $\Gamma$ is regular with valency $k$ and $\mathcal{G}$ the $1-(|E|, k, 2)$ incidence design for $\Gamma$, then $\operatorname{Aut}(\Gamma)=\operatorname{Aut}(\mathcal{G})$. The neighbourhood design of a regular graph is the 1-design formed by taking the points to be the vertices of the graph and the blocks to be the sets of neighbours of a vertex, for each vertex, i.e. regarding an adjacency matrix as an incidence matrix for the design. The line graph of a graph $\Gamma=(V, E)$ is the graph $L(\Gamma)$ with $E$ as vertex set and where adjacency is defined so that $e$ and $f$ in $E$, as vertices, are adjacent in $L(\Gamma)$ if $e$ and $f$ as edges of $\Gamma$ share a vertex in $\Gamma$.

The code of a graph $\Gamma$ over a finite field $F$ is the row span of an adjacency matrix $A$ over the field $F$, denoted by $C_{F}(\Gamma)$ or $C_{F}(A)$. The dimension of the code is the rank of the matrix over $F$, also written $\operatorname{rank}_{p}(A)$ if $F=\mathbb{F}_{p}$, in which case we will speak of the p-rank of $A$ or $\Gamma$, and write $C_{p}(\Gamma)$ or $C_{p}(A)$ for the code. It is also the code over $\mathbb{F}_{p}$ of the neighbourhood design. Similarly, if $G$ is an incidence matrix for $\Gamma, C_{p}(G)$ denotes the row span of $G$ over $\mathbb{F}_{p}$ and is the code of the design with blocks the rows of $G$, in the case that $\Gamma$ is regular.

A graph is bipartite if and only if it does not have an odd cycle.

We denote the complete graph on $n$ vertices by $K_{n}$, the complete bipartite graph on $n+m$ vertices whose partite sets have $n$ and $m$ vertices, respectively, by $K_{m, n}$, and the cycle on $n$ vertices by $C_{n}$. A triangle is the graph $K_{3}$. The graph obtained from $K_{n}$, where $n$ is even, by removing a perfect matching $M$, is denoted by $K_{n}-M$. We write $C_{n} \times K_{2}$ and $C_{n}\left[K_{2}\right]$ for the cartesian product and the lexicographic product, respectively, of $C_{n}$ and $K_{2}$. These graphs are obtained from the disjoint union of two cycles on $n$ vertices, $u_{0}, u_{1}, \ldots, u_{n-1}, u_{0}$ and $v_{0}, v_{1}, \ldots, v_{n-1}, v_{0}$ by adding the set of edges $\left\{u_{i} v_{i} \mid i=0,1, \ldots, n-1\right\}$ for $C_{n} \times K_{2}$ and $\left\{u_{i} v_{i-1}, u_{i} v_{i}, u_{i} v_{i+1} \mid i=0,1, \ldots, n-1\right\}$ for $C_{n}\left[K_{2}\right]$. We denote by $M_{n}$ the Mobius ladder with $n$ rungs, i.e., the graph obtained from $C_{n} \times K_{2}$ as defined above by replacing the edges $u_{n-1} u_{0}$ and $v_{n-1} v_{0}$ by $u_{n-1} v_{0}$ and $v_{n-1} u_{0}$. The square of the cycle $C_{n}$, denoted by $C_{n}^{2}$, is the graph obtained from the cycle $u_{0}, u_{1}, \ldots, u_{n-1}, u_{0}$ by adding the edges $u_{i} u_{i+2}$ for $i=0,1, \ldots, n-1$. All subscripts are to be taken modulo $n$. A graph is said to be trianglefree or $C_{4}$-free if it does not have a subgraph isomorphic to a $K_{3}$ or $C_{4}$, respectively.

\subsection{Edge-cuts in graphs}

If $\Gamma=(V, E)$ is a connected graph, then an edge-cut of $\Gamma$ is a subset $S \subseteq E$ such that removing the edges in $S$ renders the new graph $\Gamma-S$ disconnected. The edge-connectivity of $\Gamma$, denoted by $\lambda(\Gamma)$, is the minimum cardinality of an edge-cut of $\Gamma$. A bridge of a connected graph is an edge whose removal disconnects the graph. So $\Gamma$ has a bridge if and only if $\lambda(\Gamma)=1$.

If $\Gamma-S$ has only nontrivial components, i.e., components with at least two vertices, 
then $S$ is a restricted edge-cut. The minimal cardinality of a restricted edge-cut is the restricted edge-connectivity, denoted by $\lambda^{\prime}(\Gamma)$.

Now assume that $\Gamma=(V, E)$ is connected $k$-regular, $k \geqslant 2$.

- For $x \in V$, if $S=\{x y \mid y \in N(x)\}$, then $S$ is an edge cut, so $\lambda(\Gamma) \leqslant k$.

- For $x y \in E$, if $S=\{x z \mid z \in N(x)\} \cup\{y z \mid z \in N(y)\}-\{x y\}$, then $S$ is a restricted edge-cut, so $\lambda^{\prime}(\Gamma) \leqslant 2 k-2$.

- $\Gamma$ is maximally edge-connected if $\lambda(\Gamma)=k$, and $\Gamma$ is super- $\lambda$ if $\lambda(\Gamma)=k$ and every minimal edge-cut consists of the edges incident with some vertex.

- $\Gamma$ is maximally restricted edge-connected if $\lambda^{\prime}(\Gamma)=2 k-2$, and $\Gamma$ is super- $\lambda^{\prime}$ if $\lambda^{\prime}(\Gamma)=2 k-2$ and every minimal restricted edge-cut consists of the edges incident with some edge.

- If a graph is super- $\lambda^{\prime}$, then it is also super- $\lambda$ (see Hellwig and Volkmann [18]).

In [8], a bipartition set of a non-bipartite graph $\Gamma=(V, E)$ was defined to be a set $S \subseteq E$ such that $\Gamma-S$ contains a bipartite component. The minimum cardinality of a bipartition set of $\Gamma$ is denoted by $\lambda_{b i p}(\Gamma)$.

A restricted bipartition set is a bipartition set $S \subseteq E$ such that $\Gamma-S$ has a nontrivial bipartite component, i.e., a component that is bipartite and contains more than one vertex. So $\Gamma-S$ may contain isolated vertices, but at least one of the components of $\Gamma-S$ is a connected bipartite graph on at least two vertices. The minimum cardinality of a restricted bipartition set of $\Gamma$ is denoted by $\lambda_{b i p}^{\prime}(\Gamma)$.

Suppose $\Gamma$ is connected, non-bipartite, $k$-regular $k \geqslant 2$.

- For $x \in V$, if $S=\{x y \mid y \in N(x)\}$, then $S$ is a bipartition set, so $\lambda_{b i p}(\Gamma) \leqslant k$.

- If $|V| \geqslant 3$ and $x y \in E$, if $S=\{x z \mid z \in N(x)\} \cup\{y z \mid z \in N(y)\}-\{x y\}$, then $S$ is a restricted bipartition set, so $\lambda_{b i p}^{\prime}(\Gamma) \leqslant 2 k-2$.

- $\Gamma$ is super- $\lambda_{b i p}$ if $\lambda_{b i p}(\Gamma)=k$ and every minimum bipartition set consists of the edges incident with some vertex.

- $\Gamma$ is super- $\lambda_{b i p}^{\prime}$ if $\lambda_{b i p}^{\prime}(\Gamma)=2 k-2$ and every minimum restricted bipartition set consists of the edges incident with some edge.

- The minimum number of edges of $\Gamma$ whose removal renders the graph bipartite is denoted by $b(\Gamma)$.

The concept of super edge-connectivity was first introduced by Bauer, Suffel, Boesch, and Tindell [4] in 1981, and it has been studied extensively since. For $G$ an incidence matrix of a connected graph $\Gamma, C_{2}(G)$ is also known as the cut space of $\Gamma$, and was examined for majority-logic decoding in $[16,17]$. 


\section{Previous results}

First we state the results from Dankelmann, Key, Rodrigues [8] that give the first answers to the general questions about the codes from the incidence matrices of regular connected graphs.

The first result is well-known and quoted in [8, Result 1]:

Result 1. Let $\Gamma=(V, E)$ be a connected graph, $G$ an incidence matrix for $\Gamma$, and $C_{p}(G)$ the row-span of $G$ over $\mathbb{F}_{p}$. Then $\operatorname{dim}\left(C_{2}(G)\right)=|V|-1$. For odd $p, \operatorname{dim}\left(C_{p}(G)\right)=|V|$ if $\Gamma$ is not bipartite, and $\operatorname{dim}\left(C_{p}(G)\right)=|V|-1$ if $\Gamma$ is bipartite.

The results from [8] for binary codes and that we now wish to extend to $p$-ary codes are as follows, starting with the minimum weight and words, and then referring to a list from the literature of such graphs that are super- $\lambda$, (see [8, Result 2]):

Result 2. (Dankelmann, Key, Rodrigues [8, Theorem 1]) Let $\Gamma=(V, E)$ be a connected graph, $G$ a $|V| \times|E|$ incidence matrix for $\Gamma$. Then

1. $C_{2}(G)$ is a $[|E|,|V|-1, \lambda(\Gamma)]_{2}$ code;

2. if $\Gamma$ is super- $\lambda$, then $C_{2}(G)=[|E|,|V|-1, \delta(\Gamma)]_{2}$, and the minimum words are the rows of $G$ of weight $\delta(\Gamma)$.

For the gap in the weight-enumerator, and referring to the list in [8, Result 4]:

Result 3. [8, Theorem 4] Let $\Gamma=(V, E)$ be a connected $k$-regular graph with $|V| \geqslant 4, G$ an incidence matrix for $\Gamma, \lambda(\Gamma)=k$ and $\lambda^{\prime}(\Gamma)>k$. Let $W_{i}$ be the number of codewords of weight $i$ in $C_{2}(G)$. Then $W_{i}=0$ for $k+1 \leqslant i \leqslant \lambda^{\prime}(\Gamma)-1$, and $W_{\lambda^{\prime}(\Gamma)} \neq 0$ if $\lambda^{\prime}(\Gamma)>k+1$.

We had similar results for bipartite connected graphs for $p$ odd:

Result 4. [8, Theorem 2] Let $\Gamma=(V, E)$ be a connected bipartite graph, $G$ a $|V| \times|E|$ incidence matrix for $\Gamma$, and $p$ an odd prime. Then

1. $C_{p}(G)$ is a $[|E|,|V|-1, \lambda(\Gamma)]_{p}$ code;

2. if $\Gamma$ is super- $\lambda$, then $C_{p}(G)=[|E|,|V|-1, \delta(\Gamma)]_{p}$, and the minimum words are the non-zero scalar multiples of the rows of $G$ of weight $\delta(\Gamma)$.

Result 5. [8, Theorem 5] Let $\Gamma=(V, E)$ be a connected bipartite $k$-regular graph with $|V| \geqslant 4, G$ an incidence matrix for $\Gamma, \lambda(\Gamma)=k$ and $\lambda^{\prime}(\Gamma)>k$. Let $W_{i}$ be the number of codewords of weight $i$ in $C_{p}(G)$ where $p$ is odd. Then $W_{i}=0$ for $k+1 \leqslant i \leqslant \lambda^{\prime}(\Gamma)-1$, and $W_{\lambda^{\prime}(\Gamma)} \neq 0$ if $\lambda^{\prime}(\Gamma)>k+1$.

For non-bipartite graphs for the $p$-ary case to match Result 2 we had:

Result 6. [8, Theorem 3] Let $\Gamma=(V, E)$ be a connected graph that is not bipartite, $p$ be an odd prime, and $G$ be an incidence matrix for $\Gamma$. Then 
1. $C_{p}(G)$ is a $\left[|E|,|V|, \lambda_{b i p}(\Gamma)\right]_{p}$ code;

2. if $\Gamma$ is super- $\lambda_{\text {bip }}$ then $C_{p}(G)=[|E|,|V|, \delta(\Gamma)]_{p}$, and the minimum words are the non-zero scalar multiples of the rows of $G$ of weight $\delta(\Gamma)$.

For the previous result we used the following result, which we will use again in this paper in order to extend Result 8 below:

Result 7. [8, Proposition 1] Let $\Gamma=(V, E)$ be a connected graph that is not bipartite. Then

$$
\lambda_{\text {bip }}(\Gamma) \geqslant \min \{\lambda(\Gamma), b(\Gamma)\} .
$$

1. If $\Gamma$ is $k$-regular, $b(\Gamma) \geqslant k$ and $\lambda(\Gamma)=k$, then $\lambda_{b i p}(\Gamma)=k$.

2. If $\Gamma$ is $k$-regular and super- $\lambda$, and if $b(\Gamma)>k$ then $\Gamma$ is super- $\lambda_{\text {bip }}$.

Using the two previous results, for the non-bipartite $p$-ary case we had:

Result 8. [8, Corollary 3] Let $\Gamma=(V, E)$ be a connected $k$-regular graph that is not bipartite on $|V|=n$ vertices, $G$ an $n \times \frac{n k}{2}$ incidence matrix for $\Gamma$, and $p$ an odd prime. If

1. $k \geqslant(n+3) / 2$ and $n \geqslant 6$, or

2. $\Gamma$ is strongly regular with parameters $(n, k, \lambda, \mu)$, where

(a) $n \geqslant 7, \mu \geqslant 1,1 \leqslant \lambda \leqslant k-3$, or (b) $n \geqslant 11, \mu \geqslant 1, \lambda=0$,

then the code $C_{p}(G)$ has minimum weight $k$, and the minimum words are the non-zero scalar multiples of the rows of $G$.

In [8] we did not consider the nature of the words of weight $2 k-2$, thus all cases for that consideration are new to this paper.

The following conditions for a connected $k$-regular graph $\Gamma$ to be super- $\lambda^{\prime}$ can be found in the literature. Firstly we state the results for non-bipartite graphs, but note that in many cases the original paper contains more general statements.

Result 9. Let $\Gamma=(V, E)$ be a k-regular connected graph on $|V|=n$ vertices which is not bipartite.

(a) $\Gamma$ is super- $\lambda$ if one of the following holds:

1. $\Gamma$ is vertex-transitive and has no complete subgraph of order $k$ (Tindell [27]);

2. $\Gamma$ is edge-transitive and not a cycle (Tindell [27]);

3. $\Gamma$ has diameter at most 2 , and in addition $\Gamma$ has no complete subgraph of order $k$ (Fiol [11]);

4a. $\Gamma$ is strongly regular with parameters $(n, k, \lambda, \mu)$ with $\lambda \leqslant k-3, \mu \geqslant 1$ (from 3 above); 
4b. $\Gamma$ is distance regular and $k>2$ (Brouwer and Haemers [6]);

5. $\Gamma$ is $k$-regular and $k \geqslant \frac{n+1}{2}$ (Kelmans [19]);

6. $\Gamma$ has girth $g$, $\operatorname{diam}(\Gamma) \leqslant g-1$ if $g$ is odd and $\operatorname{diam}(\Gamma) \leqslant g-2$ if $g$ is even. (Fàbrega and Fiol [10]).

(b) $\Gamma$ is super- $\lambda^{\prime}$ if one of the following holds:

$1 a^{\prime} . \Gamma$ is vertex-transitive, has girth at least 5, and is not a cycle (Wang, [29]);

$1 b^{\prime} . \Gamma$ is vertex-transitive and

(i) not contained in $\left\{K_{n}, C_{n}, C_{m}\left[K_{2}\right], C_{m} \times K_{2}, C_{2 m}^{2}, M_{m}\right\}$ where $m=\frac{n}{2}$;

(ii) not a 4-regular vertex-transitive graph in which every vertex is contained in exactly two triangles;

(iii) does not contain a $(k-1)$-regular subgraph with $\ell$ vertices where $k \leqslant \ell \leqslant 2 k-3$;

(iv) does not contain a $(k-1)$-regular subgraph with $2 k-2$ vertices and $n \geqslant 2 k+1$;

$(v)$ does not contain a $(k-1)$-clique and is not isomorphic to a $(k+1)$-clique.

(Yang et al. [30]);

$2^{\prime} . \Gamma$ is edge-transitive, $\Gamma \notin\left\{K_{6}-M, C_{n}\right\}$ and $\Gamma$ is not the line graph of a triangle-free 3-regular edge-transitive graph (Tian and Meng [25]);

$3 a^{\prime}$. any two non-adjacent vertices of $\Gamma$ have at least three neighbours in common, and $|V| \geqslant 13$ (Wang et al. [28]);

$3 b^{\prime}$. any two non-adjacent vertices of $\Gamma$ have at least two neighbours in common, any two adjacent vertices have at most one neighbour in common, and $|V| \geqslant 10$ (Wang et al. [28]);

$4^{\prime} . \Gamma$ is strongly regular with parameters $(n, k, \lambda, \mu)$ with either $\mu \geqslant 3$ and $n \geqslant 13$, or with $\lambda \leqslant 1, \mu \geqslant 2$ and $n \geqslant 10$ (from $3 a^{\prime}, b^{\prime}$ above);

$5^{\prime}$. $\Gamma$ is $k$-regular and $k \geqslant \frac{1}{2} n+1$ (Ou and Zhang [24]);

$6^{\prime} . \Gamma$ has girth $g$, and $\operatorname{diam}(\Gamma) \leqslant g-3$ (Balbuena, Lin, Miller [3]).

The similar results for bipartite graphs:

Result 10. Let $\Gamma=(V, E)$ be a k-regular connected bipartite graph on $n$ vertices.

(a) $\Gamma$ is super- $\lambda$ if one of the following holds:

1. $\Gamma$ is half-vertex-transitive, i.e., its automorphism group acts transitively on each partite set (Tian, Meng and Liang, [26]);

2. $\Gamma$ is edge-transitive and not a cycle (Tindell [27]); 
3. $\Gamma$ has diameter 3 (or equivalently, any two vertices in the same partite set have a neighbour in common) and $k<\frac{n}{4}$ (Balbuena, Carmona, Fàbrega and Fiol [2]);

4. $\Gamma$ is $k$-regular and $k \geqslant\left\lfloor\frac{n+2}{4}\right\rfloor+1$ (Fiol [11]).

(b) $\Gamma$ is super- $\lambda^{\prime}$ if one of the following holds:

$1^{\prime} . \Gamma$ is half-vertex-transitive, $\Gamma \notin\left\{C_{n}, C_{n / 2} \times K_{2}, M_{n / 2}\right\}$, and $\Gamma$ contains no $K_{k-1, k-1}$ (Tian, Meng, and Liang [26]);

$2^{\prime} . \Gamma$ is edge-transitive, $\Gamma \neq Q_{3}$ (hypercube with $n=8, k=3$ ), and $\Gamma$ is not of girth 3 with $k=4$ and $n \geqslant 6$ (J.-X. Zhou [32]);

$3^{\prime}$. $\Gamma$ has a perfect matching and any two vertices in the same partite set have at least three common neighbours (Yuan et al. [31]);

$4^{\prime} .4(k-1)^{2} \geqslant k(n-4)-2$ if $n$ is odd, $4(k-1)^{2} \geqslant k(n-4)-1$ if $n$ is even, and $n \geqslant 22$ (Meierling and Volkmann [23]);

5'. $\Gamma$ has girth $g$, and $\operatorname{diam}(\Gamma) \leqslant g-3$ (Balbuena, Lin, Miller [3]).

\section{Words of weight $2 k-2$ in the binary codes}

Results 2 and 3 tell us about the minimum weight, the nature of the minimum words and the gap in the weight enumerator, in the binary codes $C_{2}(G)$. We now establish the nature of the words of the next weight, i.e. $2 k-2$, for the $k$-regular connected graphs that are super- $\lambda^{\prime}$. We take $k \geqslant 3$ to avoid trivial cases.

Theorem 11. Let $\Gamma=(V, E)$ be a k-regular, connected, super- $\lambda^{\prime}$ graph. Let $G$ be an incidence matrix for $\Gamma$. Let $W_{i}$ be the number of codewords of $C_{2}(G)$ of weight $i$. Then $W_{i}=0$ for $i \in\{1,2, \ldots, k-1\} \cup\{k+1, k+2, \ldots, 2 k-3\}$. Moreover,

(i) $W_{k}=|V|$ and the words of weight $k$ are the rows of $G$;

(ii) $W_{2 k-2}=|E|$ and every word of weight $2 k-2$ is a difference of two rows of $G$ corresponding to two adjacent vertices.

Proof. The nature of the minimum words and the gap in the weight enumerator follow from Results 2 and 3, since $\Gamma$ is also super- $\lambda$, as noted in Section 2.3.

Now let $w$ be a word of weight $2 k-2$, and $S=\operatorname{Supp}(w)$. Then, as shown in $[8$, Theorem 1], $\Gamma-S$ is disconnected, so $S$ is an edge-cut. Suppose the components are $W$ and $V-W$. All the edges in $S$ are between $W$ and $V-W$, and since $|S|=2 k-2, W$, and $V-W$ must have at least two vertices, and thus $S$ is a restricted edge-cut. Since its size is $\lambda^{\prime}(\Gamma)$ and $\Gamma$ is super- $\lambda^{\prime}, S$ must consist of all the edges through a pair of adjacent vertices, $x$ and $y$, excluding the edge $x y$. Thus $w=G_{x}-G_{y}$. 
Now the list of regular connected super- $\lambda^{\prime}$ graphs in Result 9 can be consulted to see which graphs this result applies to.

Example 12. In [12] uniform subset graphs were looked at and some properties deduced that we can use here. For $n>k>r \geqslant 0$, define $\Gamma=G(n, k, r)$ to be the graph with vertex set $\Omega^{\{k\}}$, the set of $k$-subsets of a set $\Omega$ of size $n$, and adjacency defined by the rule that $a, b \in \Omega^{\{k\}}$ are adjacent if $|a \cap b|=r$. The valency is $\nu=\left(\begin{array}{l}k \\ r\end{array}\right)\left(\begin{array}{l}n-k \\ k-r\end{array}\right)$. The graphs are edge-transitive (see, for example, [12]) and hence by a result quoted in $[8$, Result $4(2)]$, for them $\lambda^{\prime}(\Gamma)=2 \nu-2$. If they are super- $\lambda^{\prime}$ then they will satisfy Theorem 11. By Result $9\left(2^{\prime}\right)$, this will be so if $\nu \neq 2,4$. The graphs with $\nu=2,4$ are: $L\left(K_{3}\right)=T(3)=G(3,2,1), L\left(K_{4}\right)=T(4)=G(4,2,1)=K_{6}-M$, i.e. triangular graphs, and the odd graph $\mathcal{O}_{3}=G(7,3,0)^{1}$. Of these, $T(3)$ has valency 2 , so we exclude it; $T(4)$ has more words of weight 6 in $C_{2}(G) ; \mathcal{O}_{3}$ does satisfy the conclusions of the theorem, as does $\mathcal{O}_{2}$, the Petersen graph. Computations are with Magma $[5,7]$.

Example 13. For strongly regular graphs, Result $9\left(4^{\prime}\right)$ can be applied. For the Paley graphs $P(q)$ Theorem 11 will apply for $q>9$. For $q=9, P(9)$ has parameters $(9,4,1,2)$ and thus is not covered by that result, although it is covered by Result 2 for the minimum words and the gap in the weight enumerator. In fact there are six words of weight $2 k-2=6$ in $C_{2}(G)$ (where $G$ is an incidence matrix for $P(9)$ ) that are not from the difference of two intersecting rows. These are the words $\sum_{x \in \Delta} G_{x}$ where $\Delta$ is any of the six triangles of points in $P(9)$.

\section{Gap in the weight enumerator for $p$ odd}

Notice that from Result 5, we need only establish the gap in the weight enumerator for the non-bipartite graphs for the $p$ odd case.

In analogy to Results 3 and 5 we now have the following:

Theorem 14. Let $\Gamma=(V, E)$ be a connected $k$-regular graph with $\lambda(\Gamma)=k$ which is not bipartite, $G$ an incidence matrix for $\Gamma, \lambda_{\text {bip }}(\Gamma)=k$ and $\lambda_{b i p}^{\prime}(\Gamma)>k$. Let $p$ be an odd prime, and let $W_{i}$ be the number of codewords of $C_{p}(G)$ of weight $i$. Then $W_{i}=0$ for $i=k+1, k+2, \ldots, \lambda_{b i p}^{\prime}(\Gamma)-1$, and $W_{i}>0$ for $i=\lambda_{b i p}^{\prime}(\Gamma)$.

Proof. Let $d$ be the minimum weight of $C_{p}(G)$. By Result 6 we have $d=k$. It suffices to show that for every nonzero word $x \in C_{p}(G)$ we have $\operatorname{wt}(x)=k \operatorname{or} \operatorname{wt}(x) \geqslant \lambda_{b i p}^{\prime}(\Gamma)$, and that $C_{p}(G)$ contains a word of weight $\lambda_{b i p}^{\prime}(\Gamma)$.

Fix a nonzero word $x=\sum_{v \in V} \mu_{v} G_{v}$, and for any scalar $\alpha$ define $V_{\alpha}$ to be the set of all vertices $v$ with $\mu_{v}=\alpha$. Let $\Gamma_{x}=\Gamma(V, E-\operatorname{Supp}(x))$.

Consider a vertex $v \in V_{\alpha}$ where $\alpha \neq 0$. For every neighbour $w$ of $v$ in $\Gamma_{x}$ we have $v w \notin \operatorname{Supp}(x)$, and so $\mu_{v}+\mu_{w}=0$, i.e., $\mu_{w}=-\alpha$. For every neighbour $u$ of a neighbour $w$ of $v$ in $\Gamma_{x}$, we have $u w \notin \operatorname{Supp}(x)$ and so $\mu_{w}+\mu_{u}=0$, i.e., $\mu_{u}=\alpha$. Repeating this argument we obtain that the component of $\Gamma_{x}$ containing $v$ is bipartite, where the partite

\footnotetext{
${ }^{1}$ Frequently denoted $O_{4}$ in the literature.
} 
set containing $v$ is contained in $V_{\alpha}$, and the partite set not containing $v$ is contained in $V_{-\alpha}$. So each component of $\Gamma_{x}$ is either fully contained in $V_{\alpha} \cup V_{-\alpha}$ for some $\alpha \neq 0$ and bipartite, or is contained in $V_{0}$.

CAse 1: Every nontrivial component of $\Gamma_{x}$ is contained in $V_{0}$.

Since $x$ is not the zero-word, we have $V-V_{0} \neq \emptyset$. If $V-V_{0}$ contains only one vertex, $v$ say, then $x$ has weight $k$ and is a multiple of $G_{v}$. If $V-V_{0}$ contains at least two vertices, $v$ and $w$ say, then each of these vertices forms a component of $\Gamma_{x}$, and so every edge incident with $v$ or $w$ is in $\operatorname{Supp}(x)$. Since at most one edge is incident with both, $v$ and $w$, we have $|\operatorname{Supp}(x)| \geqslant 2 k-1>\lambda_{b i p}^{\prime}(\Gamma)$, and so $\mathrm{wt}(x)>\lambda_{b i p}^{\prime}(\Gamma)$, as desired.

CASE 2: $\Gamma_{x}$ contains a nontrivial component which is not contained in $V_{0}$.

Then $S=\operatorname{Supp}(x)$ is a restricted bipartition set and so wt $(x)=|S| \geqslant \lambda_{b i p}^{\prime}(\Gamma)$, as desired.

To see that there exists a word of weight $\lambda_{b i p}^{\prime}(\Gamma)$ consider a minimum restricted bipartition set $S$, and let $\Gamma_{1}$ be a non-trivial bipartite component of $\Gamma-S$, and let $V_{1}$ and $V_{2}$ be the partite sets of $\Gamma_{1}$. Let $x=\sum_{v \in V} \alpha_{v} G_{v}$, where $\alpha_{v}$ is defined as follows:

$$
\alpha_{v}=\left\{\begin{array}{cc}
1 & \text { if } v \in V_{1}, \\
-1 & \text { if } v \in V_{2}, \\
0 & \text { if } v \in V-\left(V_{1} \cup V_{2}\right) .
\end{array}\right.
$$

Since no proper subset of $S$ is a bipartition set, $S$ contains exactly those edges that join either two vertices of $\Gamma_{1}$ in the same partite set, or a vertex in $\Gamma_{1}$ to a vertex not in $\Gamma_{1}$. Hence $S=\operatorname{Supp}(x)$, and so wt $(x)=|S|=\lambda_{b i p}^{\prime}(\Gamma)$.

Recall that it has been shown by Hellwig and Volkmann [18] that if a graph is super$\lambda^{\prime}$, then it is also super- $\lambda$. The following lemma shows that a similar statement holds for super- $\lambda_{b i p}^{\prime}$ graphs.

Lemma 15. Let $\Gamma$ be a connected, non-bipartite, $k$-regular graph, where $k \geqslant 3$.

(i) If $\lambda_{b i p}^{\prime}(\Gamma) \geqslant k$ then $\lambda_{b i p}(\Gamma)=k$.

(ii) If $\lambda_{b i p}^{\prime}(\Gamma)>k$ then $\Gamma$ is super- $\lambda_{b i p}$.

(iii) If $\Gamma$ is super- $\lambda_{\text {bip }}^{\prime}$, then $\Gamma$ is also super- $\lambda_{\text {bip }}$.

Proof. Let $S \subset E(\Gamma)$ be a minimum bipartition set and let $\Gamma_{1}$ be a bipartite component of $\Gamma-S$ of largest order. We first note that if $\Gamma-S$ is connected, then $S$ is a restricted bipartition set, and so $\lambda_{b i p}^{\prime}(\Gamma)=|S|=\lambda_{b i p}(\Gamma) \leqslant k$.

(i) Let $\lambda_{b i p}^{\prime}(\Gamma) \geqslant k$. If $\Gamma-S$ is connected, then it follows from the above that $\lambda_{b i p}(\Gamma)=k$, so we may assume that $\Gamma-S$ is disconnected. If $\Gamma_{1}$ consists of a single vertex, then $S$ contains the edges incident with this vertex, and so we have $\lambda_{b i p}(\Gamma)=|S| \geqslant k$, and if $\Gamma_{1}$ is a nontrivial component, then $S$ is a restricted bipartition set, and so $|S| \geqslant \lambda_{b i p}^{\prime}(\Gamma) \geqslant k$. Since always $\lambda_{\text {bip }}(\Gamma) \leqslant k$, we have $\lambda_{b i p}(\Gamma)=k$ in all cases.

(ii) Since $\lambda_{b i p}^{\prime}(\Gamma)>k$, it follows from the above that $\Gamma-S$ is disconnected. Then $\Gamma_{1}$ 
consists of a single vertex, since otherwise $S$ is a restricted bipartition set and we obtain $\lambda_{b i p}(\Gamma)=|S| \geqslant \lambda_{b i p}^{\prime}(\Gamma)>k$, contradicting $\lambda_{b i p}(\Gamma) \leqslant k$. Let $v$ be the vertex in $\Gamma_{1}$. Then since $S$ is a minimal bipartition set, it follows that only the edges incident with $v$ are in $S$. Since $S$ is an arbitrary minimal bipartition set, this implies that $\Gamma$ is super- $\lambda_{\text {bip }}$.

(iii) This statement is a direct consequence of (ii), since for every $k$-regular super- $\lambda_{b i p}^{\prime}$ graph with $k \geqslant 3$ we have $\lambda_{b i p}^{\prime}(\Gamma)>k$.

\section{Words of weight $2 k-2$ for $p$ odd}

Now we show that the words of weight $2 k-2$ in the $p$-ary case for $p$ odd are also, for a wide variety of connected regular graphs, as expected. Notice that in the bipartite case we can prove the following in precisely the same way as in Theorem 11 for the binary case:

Theorem 16. Let $\Gamma=(V, E)$ be a k-regular, bipartite, connected, super- $\lambda^{\prime}$ graph. Let $G$ be an incidence matrix for $\Gamma, p$ an odd prime. Let $W_{i}$ be the number of codewords of $C_{p}(G)$ of weight $i$. Then $W_{i}=0$ for $i \in\{1,2, \ldots, k-1\} \cup\{k+1, k+2, \ldots, 2 k-3\}$. Moreover,

(i) $W_{k}=(p-1)|V|$ and the words of weight $k$ are the scalar multiples of the rows of $G$;

(ii) $W_{2 k-2}=(p-1)|E|$ and every word of weight $2 k-2$ is a scalar multiple of the difference of two rows of $G$ corresponding to two adjacent vertices.

Consulting the list of families of graphs that are $k$-regular, bipartite, connected, and super- $\lambda^{\prime}$, will tell us which families satisfy the theorem. Some of these can be found in Result 10 .

Thus from now on we concentrate on the non-bipartite, $p$ odd, case.

Lemma 17. Let $\Gamma$ be a connected non-bipartite graph and let $S$ be a minimum restricted bipartition set. Then $\Gamma-S$ contains exactly one bipartite component.

Proof. Let $\Gamma_{1}$ be a non-trivial bipartite component of $\Gamma-S$. Suppose by way of contradiction that $\Gamma-S$ has another bipartite component, $\Gamma_{2}$ say. Since $S$ is minimal, every edge of $S$ is incident with a vertex $\Gamma_{1}$. Since $\Gamma$ is connected it follows that there exists an edge $e \in S$ joining a vertex in $\Gamma_{1}$ to a vertex in $\Gamma_{2}$. We claim that $S^{\prime}=S-\{e\}$ is a restricted bipartition set. Clearly, $\left(\Gamma_{1} \cup \Gamma_{2}\right)+e$ is a nontrivial component of $\Gamma-S^{\prime}$. This component does not contain an odd cycle since neither $\Gamma_{1}$ nor $\Gamma_{2}$ contain an odd cycle, and there is no cycle containing $e$ since $e$ is a bridge of $\left(\Gamma_{1} \cup \Gamma_{2}\right)+e$. Hence it is also bipartite, and so $S^{\prime}$ is a smaller restricted bipartition set, a contradiction to the minimality of $S$. 
Theorem 18. Let $\Gamma=(V, E)$ be a k-regular, connected, non-bipartite, super- $\lambda_{b i p}^{\prime}$ graph. Let $G$ be an incidence matrix for $\Gamma$. Let $p$ be an odd prime, and let $W_{i}$ be the number of codewords of $C_{p}(G)$ of weight $i$. Then $W_{i}=0$ for $i \in\{1,2, \ldots, k-1\} \cup\{k+1, k+$ $2, \ldots, 2 k-3\}$. Moreover,

(i) $W_{k}=(p-1)|V|$ and the words of weight $k$ are the scalar multiples of the rows of $G$;

(ii) $W_{2 k-2}=(p-1)|E|$, and every word of weight $2 k-2$ is a scalar multiple of a difference of two rows of $G$ corresponding to two adjacent vertices.

Proof. (i) It follows from [8, Theorem 3] that $W_{1}=W_{2}=\ldots=W_{k-1}=0$ and the words of weight $k$ are the scalar multiples of rows of $G$. Hence we have $W_{k}=(p-1) n$.

(ii) It follows from Theorem 14 and the fact that $\lambda_{b i p}^{\prime}(\Gamma)=2 k-2$ that $W_{k+1}=W_{k+2}=$ $\ldots=W_{2 k-3}=0$ and $W_{2 k-2}>0$. Consider a word $x \in C_{p}(G)$ of weight $2 k-2$. We use the same notation as in the proof of Theorem 14. If every nontrivial component of $\Gamma_{x}$ is contained in $V_{0}$, then, as in the proof of Theorem 14,w(x) either equals $k$ or is at least $2 k-1$, so this case cannot occur. Hence $\Gamma_{x}$ has a nontrivial component $\Gamma_{1}$ not contained in $V_{0}$. As in the proof of Theorem $14, \Gamma_{1}$ is bipartite, i.e., $\operatorname{Supp}(x)$ is a restricted bipartition set of cardinality wt $(x)=2 k-2$. Since $\Gamma$ is super- $\lambda_{b i p}^{\prime}$, this implies that $\operatorname{Supp}(x)$ consists of the edges incident with the vertices of an edge $u v$, except the edge $u v$ itself, and that $\Gamma_{1}$ contains only the vertices $u$ and $v$.

By Lemma 17, $\Gamma_{1}$ is the only bipartite component of $\Gamma_{x}$. Therefore, $\mu_{w}=0$ for all vertices $w$ not in $\Gamma_{1}$, and furthermore $\mu_{u}=-\mu_{v}$. Hence $x=\alpha G_{u}-\alpha G_{v}$ for some scalar $\alpha \neq 0$, and so $x$ is a scalar multiple of the difference of two rows of $G$ corresponding to adjacent vertices.

We will frequently make use of the following proposition in order to show that a graph is super- $\lambda_{b i p}$.

Proposition 19. Let $\Gamma$ be a connected, $k$-regular graph that is not bipartite. Then

(a) $\lambda_{b i p}^{\prime}(\Gamma) \geqslant \min \left\{\lambda^{\prime}(\Gamma), b(\Gamma)\right\}$.

(b) If $\lambda^{\prime}(\Gamma)=2 k-2$ and $b(\Gamma) \geqslant 2 k-2$, then $\lambda_{b i p}^{\prime}(\Gamma)=2 k-2$.

(c) If $\Gamma$ is super $-\lambda^{\prime}$ and $b(\Gamma)>2 k-2$, then $\Gamma$ is super- $\lambda_{b i p}^{\prime}$.

Proof. (a) Let $S$ be a set of cardinality $\lambda_{b i p}^{\prime}(\Gamma)$ such that $\Gamma-S$ contains a nontrivial bipartite component $\Gamma_{1}$. If $\Gamma_{1}$ contains all vertices of $\Gamma$, then $\Gamma-S$ is bipartite, and so $|S| \geqslant b(\Gamma) \geqslant \min \left\{\lambda^{\prime}(\Gamma), b(\Gamma)\right\}$. Hence we may assume that $\Gamma_{1}$ does not contain all vertices of $\Gamma$. By Lemma $17, \Gamma-S$ contains no bipartite component other than $\Gamma_{1}$, and in particular no trivial component. Hence every component of $\Gamma-S$ is non-trivial, and so $S$ is a restricted edge-cut, implying that $|S| \geqslant \lambda^{\prime}(\Gamma)$. Hence (a) follows.

(b) The statement in (b) is a direct consequence of (a).

(c) Let $S$ be as in (a). Since $|S|=\lambda_{b i p}^{\prime}(\Gamma) \leqslant 2 k-2<b(\Gamma)$, as above, $S$ is a restricted edge-cut, and $S$ contains at most $2 k-2$ edges. Since $\Gamma$ is super- $\lambda^{\prime}$, we have $|S|=2 k-2$, and $S$ consists of the edges incident with an edge of $\Gamma$. Hence $\Gamma$ is super- $\lambda_{b i p}^{\prime}$. 
We now give sufficient conditions for a graph to be super- $\lambda_{b i p}^{\prime}$ or super- $\lambda_{b i p}$.

We first consider conditions on the vertex degrees. In [8, Corollary 3] it was shown that for a $k$-regular non-bipartite graph on $n \geqslant 6$ vertices with $k \geqslant \frac{n+3}{2}$ we always have $\lambda_{\text {bip }}(\Gamma)=k$. Below we show that $k \geqslant \frac{n+5}{2}$ guarantees the non-bipartite graph to be super- $\lambda_{b i p}^{\prime}$. In the proof we use the well-known fact that a bipartite graph on $n$ vertices has at most $\frac{n^{2}}{4}$ edges.

Proposition 20. Let $\Gamma=(V, E)$ be a connected non-bipartite $k$-regular graph with $|V|=n$ and $k \geqslant \frac{n+5}{2}$ and $n \geqslant 13$. Then $\Gamma$ is super- $\lambda_{\text {bip }}^{\prime}$.

Proof. We note that $\Gamma$ is super- $\lambda^{\prime}$ by Result $9\left(3 a^{\prime}\right)$, so, by Proposition $19(\mathrm{c})$ it suffices to show that $b(\Gamma)>2 k-2$.

Since a bipartite graph on $n$ vertices has at most $\frac{n^{2}}{4}$ edges, we have

$$
b(\Gamma) \geqslant|E(\Gamma)|-\frac{1}{4} n^{2}=\frac{1}{2} k n-\frac{1}{4} n^{2}>2 k-2 .
$$

It is easy to verify that the last inequality holds provided $k \geqslant \frac{n+5}{2}$ and $n \geqslant 13$.

Note that the condition $k \geqslant \frac{n+5}{2}$ is best possible to guarantee that $\Gamma$ is super- $\lambda_{b i p}^{\prime}$. To see this let $n$ be even and let $\Gamma$ be the graph obtained from a complete bipartite graph $K_{n / 2, n / 2}$ with partite sets $U$ and $W$ by adding the edges of two cycles, one through the vertices of $U$, and the other through the vertices of $W$. Then $\Gamma$ is $k$-regular with $k=\frac{n+4}{2}$. It is easy to verify that $\lambda_{b i p}^{\prime}(\Gamma)=b(\Gamma)=n=2 k-4$.

We note further that the condition $k \geqslant \frac{n+3}{2}$ given in [8] is best possible to guarantee that $\lambda_{b i p}(\Gamma)=k$. To see this let $n \equiv 0(\bmod 4)$ and let $\Gamma$ be the graph obtained from a complete bipartite graph $K_{n / 2, n / 2}$ by adding the edges of a perfect matching. Then $\Gamma$ is $k$-regular with $k=\frac{n+2}{2}$. It is easy to verify that $\lambda_{b i p}^{\prime}(\Gamma)=b(\Gamma)=\frac{n}{2}=k-1$.

We now give lower bounds on $b(\Gamma)$ which will be needed when we consider strongly regular graph, edge-transitive graphs and vertex transitive graphs. While several results for upper bounds on $b(\Gamma)$ are known (usually stated as lower bounds on the maximal number of edges in a bipartite subgraph of $\Gamma$ ), little appears to be known regarding lower bounds on $b(\Gamma)$.

Lemma 21. Let $a \geqslant 3$ be odd, and let $\Gamma$ be a graph of order $n$ containing a cycle of length a.

(i) If $\Gamma$ is $k$-regular graph and every edge of $\Gamma$ is on the same number of cycles of length $a$, then $b(\Gamma) \geqslant \frac{n k}{2 a}$.

(ii) If every vertex of $\Gamma$ is on the same number of cycles of length $a$, then $b(\Gamma) \geqslant \frac{n}{a}$.

Proof. (i) Let each edge of $\Gamma$ be contained in $t$ cycles $C_{a}$, where $t>0$. Then the total number of cycles $C_{a}$ in $\Gamma$ equals $\frac{|E| t}{a}=\frac{n k t}{2 a}$. Now removing an edge destroys at most $t$ cycles $C_{a}$. Hence, if $S \subseteq E$ is a set of $b(\Gamma)$ edges such that $\Gamma-S$ is bipartite, $|S| t$ is at least the total number of cycles $C_{a}$. Hence $|S| t \geqslant \frac{n k t}{2 a}$ and thus $b(\Gamma)=|S| \geqslant \frac{n k}{2 a}$. 
(ii) Let each vertex of $\Gamma$ be contained in $t$ cycles of length $a$, where $t>0$. Then the total number of cycles $C_{a}$ in $\Gamma$ equals $\frac{n t}{a}$. Consider an arbitrary edge $u v$. Each cycle through $u v$ also goes through $u$, so there are at most $t$ cycles through $u v$, i.e., removing $u v$ destroys at most $t$ cycles $C_{a}$. As above, $|S| t \geqslant \frac{n t}{a}$ and thus $b(\Gamma)=|S| \geqslant \frac{n}{a}$.

Now consider strongly regular graphs.

Theorem 22. Let $\Gamma=(V, E)$ be a strongly regular graph with parameters $(n, k, \lambda, \mu)$ that is not bipartite. Then $\Gamma$ is super- $\lambda_{\text {bip }}^{\prime}$ if one of the following conditions hold:

(i) $\lambda \geqslant 1, \mu \geqslant 3, n \geqslant 13$; (ii) $\lambda=1, \mu \geqslant 2, n \geqslant 10$; (iii) $\lambda=0, \mu \geqslant 2, n \geqslant 19$.

Proof. Under the conditions given in the statement $\Gamma$ is super- $\lambda^{\prime}$ by Result $9\left(4^{\prime}\right)$. Hence, by Proposition 19 it suffices to show that $b(\Gamma)>2 k-2$.

First suppose $\lambda \geqslant 1$. Then every edge is on exactly $\lambda \geqslant 1$ triangles. Applying Lemma 21 (i) with $a=3$, we get that $b(\Gamma) \geqslant \frac{n k}{6}>2 k-2$, i.e. $n k>12 k-12$, which is true for $n \geqslant 11$, and thus for $n \geqslant 13$ to give $(i)$.

If $\lambda=1$ and $\mu \geqslant 2$, then $\Gamma$ still has triangles, so if $n \geqslant 11$ the conditions are satisfied. If $n=10$ then by Equation $(1),(9-k) \mu=k(k-2)$ and there are no solutions with $0<\mu<k<8$ of this, so $($ ii $)$ is proved.

If $\lambda=0$ then it is easy to show that $\Gamma$ has 5 -cycles, and that every edge is on the same number of 5 -cycles. Hence, by Lemma 21 we have $b(\Gamma) \geqslant \frac{n k}{10}>2 k-2$, i.e. $n k>20 k-20$ true for $n \geqslant 19$, and thus (iii) is proved.

It was noted in [8] that for vertex transitive graphs $\lambda_{b i p}(\Gamma)$ does not necessarily equal $k$, and the same holds true for edge-transitive and arc-transitive graphs. Below we show that under very mild additional assumptions vertex- or edge-transitive graphs do not only satisfy $\lambda_{b i p}(\Gamma)=k$, but are also super- $\lambda_{b i p}^{\prime}$.

We first consider edge-transitive graphs. In the proof of the following theorem we make use of the well known fact that a shortest odd cycle in a graph is always a geodesic subgraph, i.e., the distance between two vertices in such a cycle is equal to the distance between the two vertices in the graph $\Gamma$. We further use bounds on the radius of graphs. The radius, $\operatorname{rad}(\Gamma)$, is defined as the smallest among the eccentricities of the vertices, where the eccentricity of a vertex $v$ is the largest of the distances between $v$ and all other vertices in $\Gamma$.

The following bound can be found in [9].

Result 23. Let $\Gamma$ be a connected graph of order $n$ and minimum degree $\delta$. If $\Gamma$ contains no 4-cycle, then $\operatorname{rad}(\Gamma) \leqslant 5 n / 2\left(\delta^{2}-2\lfloor\delta / 2\rfloor+1\right)$.

Lemma 24. Let $\Gamma$ be a connected $k$-regular, edge-transitive, triangle-free graph which is not bipartite. Then

(a) if $k \geqslant 5$ then $b(\Gamma)>k ; \quad(b)$ if $k \geqslant 7$ then $b(\Gamma)>2 k-2$. 
Proof. Let $C$ be an odd cycle of shortest length, $g_{0} \geqslant 5$. We first derive a lower bound on $n$ in terms of $g_{0}$ and $k$. Let $S=\cup_{v \in V(C)} N(v)$. Every vertex of $\Gamma$ is adjacent to at most two vertices of $C$ since a vertex adjacent to three or more vertices of $C$ would produce a triangle or a shorter odd cycle. Hence no vertex of $\Gamma$ is in more than two sets $N(v)$, $v \in V(C)$, and so

$$
n \geqslant|S| \geqslant \frac{1}{2} \sum_{v \in V(C)}|N(v)|=\frac{1}{2} g_{0} k .
$$

Applying Lemma 3 we get

$$
b(\Gamma) \geqslant \frac{n k}{2 g_{o}} \geqslant \frac{k^{2}}{4}
$$

The right hand side of the last inequality is greater than $k$ if $k \geqslant 5$, and greater than $2 k-2$ if $k \geqslant 7$.

Theorem 25. Let $\Gamma=(V, E)$ be a connected $k$-regular $(k \geqslant 3)$, edge-transitive graph which is not bipartite, nor a line graph of a 3-regular, triangle-free, edge-transitive, 2path-transitive graph. Let $|V|=n$.

1. If $\Gamma$ contains triangles then

(i) if $n \geqslant 7$ then $\Gamma$ is super- $\lambda_{b i p} ; \quad$ (ii) if $n \geqslant 11$ then $\Gamma$ is super- $\lambda_{\text {bip }}^{\prime}$.

2. If $\Gamma$ is triangle-free, then

(iii) if $k \geqslant 5$ then $\Gamma$ is super- $\lambda_{\text {bip }} ; \quad($ iv $)$ if $k \geqslant 7$ then $\Gamma$ is super- $\lambda_{\text {bip }}^{\prime}$.

Proof. First use Result $9\left(2^{\prime}\right)$ to show that $\Gamma$ is super- $\lambda^{\prime}$ : if $\Gamma$ is triangle-free then it cannot be $K_{6}-M$, nor a line graph, since for $k \geqslant 3$ the line graph will have triangles; if $\Gamma$ has triangles and $n \geqslant 7$ then $\Gamma$ is not $K_{6}-M$, and is not the line graph of a 3-regular graph as described in Result $9\left(2^{\prime}\right)$, in which case $k=4$. Since this family is excluded, Result $9\left(2^{\prime}\right)$ is satisfied, and so by Result 7 and Proposition 19 it suffices to show that $b(\Gamma)>k$ for $(i)$ and $(i i i)$, and $b(\Gamma)>2 k-2$ for $(i i)$ and $(i v)$.

If $\Gamma$ contains triangles, then every edge is on the same number of triangles. Hence, by Lemma 21 we have $b(\Gamma) \geqslant \frac{n k}{6}$, and thus $b(\Gamma)>k$ for $n>6$ and $b(\Gamma)>2 k-2$ for $n \geqslant 11$, implying (i) and (ii).

If $\Gamma$ is triangle-free, that $b(\Gamma)>k$ and $b(\Gamma)>2 k-2$ for the stated values of $k$ follows from Lemma 24.

The above theorem shows that regular edge-transitive graphs of sufficiently large vertex-degree are always super- $\lambda_{b i p}^{\prime}$. That this is not necessarily the case for edge-transitive graphs with small vertex-degrees, even if we assume arc-transitivity, can be seen from the following examples of 4- and 6-regular arc-transitive graphs for which $\lambda_{b i p}^{\prime}(\Gamma)<2 k-2$.

Example 26. Let $n \geqslant 3$ be an odd integer, and let $a \in\{2,3\}$. Consider the graph $\Gamma=C_{n}\left[\overline{K_{a}}\right]$, i.e., the graph with vertex set $\bigcup_{i=1}^{n} U_{i}$, where the $U_{i}$ are disjoint sets of cardinality $a$, with all $a^{2}$ edges between $U_{i}$ and $U_{i+1}$, where the indices are taken modulo 
$n$. Hence $\Gamma$ is edge-transitive and $k$-regular with $k=2 a$. It is easy to verify that the set of all edges between $U_{n}$ and $U_{1}$ is a minimum bipartition set, so $\lambda_{b i p}^{\prime}(\Gamma)=a^{2}<2 k-2=4 a-2$ for $a \in\{2,3\}$.

We now consider vertex-transitive graphs.

Lemma 27. Let $\Gamma$ be a connected $k$-regular, vertex-transitive graph with no 4-cycles, that is not bipartite. Then

(a) if $k \geqslant 7$ then $b(\Gamma)>k ; \quad(b)$ if $k \geqslant 12$ then $b(\Gamma)>2 k-2$.

Proof. Let $C$ be an odd cycle of shortest length, $g_{o}$, and let $v$ be a vertex on $C$. Since $\Gamma$ is vertex-transitive, the eccentricity of $v$ equals the radius of $\Gamma$. Then the two vertices, $u$ and $w$, opposite $v$ in $C$ are at distance $\frac{g_{o}-1}{2}$ from $v$ in $C$. Since $C$ is an isometric subgraph of $\Gamma$, we have

$$
\frac{g_{o}-1}{2}=d_{C}(v, w)=d_{\Gamma}(v, w) \leqslant \operatorname{rad}(\Gamma) \leqslant \frac{5 n}{2\left(\delta^{2}-2\lfloor\delta / 2\rfloor+1\right)}
$$

where the last inequality follows from Proposition 23. Dropping the floors in the last fraction, we obtain that

$$
g_{o} \leqslant \frac{5 n+k^{2}-k+1}{k^{2}-k+1}
$$

Applying Lemma 21, we get

$$
b(\Gamma) \geqslant \frac{n}{g_{o}} \geqslant \frac{n\left(k^{2}-k+1\right)}{5 n+k^{2}-k+1} .
$$

The right hand side of the last inequality is increasing in $n$ for fixed $k$. Since $\Gamma$ has no 4-cycles, we have $n \geqslant k^{2}-2\lfloor k / 2\rfloor+1$. (This well-known inequality follows from the fact that a vertex of $\Gamma, v$ say, has $k$ neighbours, and the neighbourhoods of any two neighbours of $v$ have no vertex in common other than $v$, since otherwise $v$ would be on a 4 -cycle. Note that a neighbour of $v$ may be adjacent to at most one other neighbour of $v$, which means that at most $2\lfloor k / 2\rfloor$ neighbours of $v$ are adjacent to another neighbour of $v$.) Substituting this for $n$ yields, after a simple calculation, that $b(\Gamma)>k$ for $k \geqslant 7$, and $b(\Gamma)>2 k-2$ for $k \geqslant 12$.

Theorem 28. Let $\Gamma$ be a connected $k$-regular, vertex-transitive graph with no 4-cycles, that is not bipartite. Then

(i) if $k \geqslant 7$ then $\Gamma$ is super- $\lambda_{\text {bip }} ; \quad$ (ii) if $k \geqslant 12$ then $\Gamma$ is super- $\lambda_{\text {bip }}^{\prime}$.

Proof. For $(i)$, from Result $9(1), \Gamma$ is super- $\lambda$ since $\Gamma$ does not contain $K_{7}$ as a subgraph. By Lemma 27(a), if $k \geqslant 7$ then $b(\Gamma)>k$. Hence, by Result $7, \Gamma$ is super- $\lambda_{b i p}$.

For $(i i)$, take $k \geqslant 12$ so that by Lemma $27(b), b(\Gamma)>2 k-2$. If $\Gamma$ has no triangles then by Result $9\left(1 a^{\prime}\right), \Gamma$ is super- $\lambda^{\prime}$. Since $b(\Gamma)>2 k-2$, it follows by Proposition 19(c) that $\Gamma$ is super- $\lambda_{b i p}^{\prime}$. 
If $\Gamma$ has triangles, we must use Result $9\left(1 b^{\prime}\right)$. Since $k \geqslant 12$, and $\Gamma$ has no 4 -cycles, $\Gamma$ is not in $(i)$ or $(i i)$ of Result $9\left(1 b^{\prime}\right)$. Suppose Result $9\left(1 b^{\prime}\right)(i i i)$ or $(i v)$ that $\Gamma$ has a $(k-1)$-regular subgraph $\Delta=(W, F)$ on $\ell$ vertices where $k \leqslant \ell \leqslant 2 k-2$. Let $u \in W$ and $N$ be the set of $k-1$ neighbours of $u$. If $U=W-(N \cup\{u\})$, then $|U|=\ell-k \leqslant k-2$. Clearly $\ell=k$ would imply $\Delta=K_{k}$, which we cannot have. So $U \neq \emptyset$. Any $v \in U$ has $k-1$ neighbours in $\Delta$, at most $k-3$ of which can be in $U$; uv is not an edge, so $v$ has at least two neighbours $x, y \in N$. Then $(u, x, v, y)$ is a 4 -cycle in $\Gamma$, which we have excluded. Thus $\Gamma$ is not of this type. That $\Gamma$ cannot be of type Result $9\left(1 b^{\prime}\right)(v)$ is immediate since a $(k-1)$-clique where $k \geqslant 12$ would have 4 -cycles. Hence $\Gamma$ is super- $\lambda^{\prime}$, and thus super- $\lambda_{b i p}^{\prime}$ since $b(\Gamma)>2 k-2$.

We note that for $k$-regular vertex-transitive graphs the inequality $\lambda_{b i p}(\Gamma) \geqslant k$ does not hold in general if 4-cycles are present, even for arbitrarily large vertex degrees. To see this consider the following example.

Example 29. Let $a, b$ be integers greater than $1, a$ even and $b$ odd, and let $\Gamma^{1}, \Gamma^{2}, \ldots, \Gamma^{b}$ be disjoint copies of the complete bipartite graph $K_{a, a}$. Let $U_{i}=\left\{u_{1}^{i}, u_{2}^{i}, \ldots, u_{a}^{i}\right\}$ and $W_{i}=\left\{w_{1}^{i}, w_{2}^{i}, \ldots, w_{a}^{i}\right\}$ be the partite sets of $\Gamma^{i}$. Let $\Gamma$ be the graph obtained from the union of the $\Gamma^{i}$ by adding the edges $u_{a / 2+j}^{i} w_{j}^{i+1}$ and $w_{a / 2+j}^{i} u_{j}^{i+1}, j=1,2, \ldots, a / 2$, between $\Gamma^{i}$ and $\Gamma^{i+1}$ for $i=1,2, \ldots, b-1$, and the edges $u_{a / 2+j}^{b} u_{j}^{1}$ and $w_{a / 2+j}^{b} w_{j}^{1}, j=1,2, \ldots, a / 2$ between $\Gamma^{b}$ and $\Gamma^{1}$. It is easy to verify that $\Gamma$ is $(a+1)$-regular and vertex-transitive. Clearly, the graph $\Gamma-\left\{u_{a / 2+j}^{b} u_{j}^{1}, w_{a / 2+j}^{b} w_{j}^{1} \mid j=1,2, \ldots, a / 2\right\}$ is bipartite with partite sets $\bigcup U_{i}$ and $\bigcup W_{i}$, but $\Gamma$ is not bipartite. Since removing the set $\left\{u_{a / 2+j}^{b} u_{j}^{1}, w_{a / 2+j}^{b} w_{j}^{1} \mid j=\right.$ $1,2, \ldots, a / 2\}$ renders $\Gamma$ bipartite, we have $b(\Gamma) \leqslant\left|\left\{u_{a / 2+j}^{b} u_{j}^{1}, w_{a / 2+j}^{b} w_{j}^{1} \mid j=1, \ldots, a / 2\right\}\right|=$ $a$. Hence $\Gamma$ is $(a+1)$-regular, but $\lambda_{b i p}(\Gamma) \leqslant b(\Gamma) \leqslant a$.

Corollary 30. Let $\Gamma$ be a connected, $k$-regular graph on $n$ vertices that is not bipartite, and let $p$ be an odd prime. Let $G$ be an incidence matrix for $\Gamma$. Then $\operatorname{dim}\left(C_{p}(G)\right)=n$ and if any one of the following holds:

1. $k \geqslant \frac{n+5}{2}$ and $n \geqslant 13$;

2. $\Gamma$ is strongly regular with parameters $(n, k, \lambda, \mu)$ with

(i) $\lambda \geqslant 1, \mu \geqslant 3, n \geqslant 13$; or (ii) $\lambda=1, \mu \geqslant 2, n \geqslant 10$; or (iii) $\lambda=0, \mu \geqslant 2$, $n \geqslant 19$;

3. $\Gamma$ is edge-transitive, $k \geqslant 3$ and

(i) $\Gamma$ contains triangles and $n \geqslant 11$, or $(i i) \Gamma$ is triangle-free and $k \geqslant 9$;

4. $\Gamma$ is vertex-transitive, contains no 4 -cycles, and $k \geqslant 12$,

then the minimum weight of $C_{p}(G)$ is $k$, the words of weight $k$ are precisely the scalar multiples of the rows of $G$, there are no words of weight $i$ such that $k<i<2 k-2$, and the words of weight $2 k-2$ are precisely the scalar multiples of the differences of two rows of $G$ corresponding to two adjacent vertices of $\Gamma$. 
Proof. Using Theorem 18: (1) follows from Proposition 20; (2) follows from Theorem 22; (3) follows from Theorem 25; (4) follows from Theorem 28.

These conditions can be weakened somewhat if only the minimum weight and the nature of the minimum words is required, so that Result 8 could be extended to the following:

Corollary 31. Let $\Gamma$ be a connected, k-regular graph on $n$ vertices that is not bipartite, and let $p$ be an odd prime. Let $G$ be an incidence matrix for $\Gamma$. Then $\operatorname{dim}\left(C_{p}(G)\right)=n$ and if any one of the following holds:

1. $k \geqslant \frac{n+3}{2}$ and $n \geqslant 6$;

2. $\Gamma$ is strongly regular with parameters $(n, k, \lambda, \mu)$ where (a) $n \geqslant 7, \mu \geqslant 1,1 \leqslant \lambda \leqslant k-3$, or (b) $n \geqslant 11, \mu \geqslant 1, \lambda=0$;

3. $\Gamma$ is edge-transitive, $k \geqslant 5$;

4. $\Gamma$ is vertex-transitive, $k \geqslant 7$, and $\Gamma$ contains no complete subgraph of order $k$, then the minimum weight of $C_{p}(G)$ is $k$ and the words of weight $k$ are precisely the scalar multiples of the rows of $G$.

Proof. The first two cases are from Result 8.

For (3), if $\Gamma$ is edge-transitive, and $k \geqslant 3$ then by Result 9 (2) it is super- $\lambda$, so using Result 7 , if we can show that $b(\Gamma)>k$ then we will have $\Gamma$ super- $\lambda_{\text {bip }}$ and hence we can use Result 6. From Lemma 24 we see this is true if $k \geqslant 5$.

For (4), if $\Gamma$ is vertex-transitive, with no complete subgraph of order $k$, then by Result 9 (1), it is super- $\lambda$, so using Result 7, if we can show that $b(\Gamma)>k$ then we will have $\Gamma$ super- $\lambda_{b i p}$ and hence we can use Result 6 . From Lemma 27 this is true for $k \geqslant 7$.

Example 32. The Hamming graph $H(d, q)$, where $q, d \geqslant 2$, has for vertices the $q^{d}$ words of length $d$ over an alphabet of $q$ elements, and where two vertices are adjacent if their words differ in exactly one position. For all $d, q H(d, q)$ is $k$-regular with $k=d(q-1)$, and it is edge-transitive. It is bipartite if $q=2$, and for $d \geqslant 4 H(d, 2)=Q_{d}$ will satisfy Theorem 16, using Result $10\left(2^{\prime}\right)$. For $d=3, H(3,2)=Q_{3}$ and for all $p$ there are words of weight 4 that are not from the difference of two intersecting rows. For $q \geqslant 3, H(d, q)$ contains triangles, and so the conclusion of Corollary 30(3) holds for $H(d, q)$ for $p$ odd if $q^{d} \geqslant 11$, i.e. all $d \geqslant 2$ and $q \geqslant 3$ apart from $d=2, q=3$. Using Magma $H(2,3)$ was shown not to satisfy the conclusion for all $p$. Note that for the binary case it is excluded by Result $9\left(2^{\prime}\right)$ since $H(2,3)=L\left(K_{3,3}\right)$. 
Example 33. The square lattice graph $L_{2}(m)$ and the triangular graph $T(m)(G(m, 2,1)$ in the notation of Example 12) are edge-transitive, both are line graphs and thus contain triangles, and so the conclusion of Corollary 30(3) holds for these graphs if they have at least 11 vertices, i.e., for $L_{2}(m)$ if $m \geqslant 4$, and for $T(m)$ if $m \geqslant 6$. Both graphs are also strongly regular: $L_{2}(m)$ is an $\left(m^{2}, 2(m-1), m-2,2\right)$ and $T(m)$ is $\left(\left(\begin{array}{c}m \\ 2\end{array}\right), 2(m-2), m-2,4\right)$, and neither $L_{2}(3)$ nor $T(5)$ satisfy the requirements of Corollary 30(2). Notice that for $G$ an incidence matrix for $T(5), C_{p}(G)$, for any $p$ odd, has words of weight 10 that are not the difference of two rows. These can be constructed from two disjoint 5-cycles, for example $c_{1}=(\{1,2\},\{2,3\},\{3,4\},\{4,5\},\{5,1\})$ and $c_{2}=(\{1,3\},\{3,5\},\{5,2\},\{2,4\},\{4,1\})$, and then $w=\sum_{u \in c_{1}} G_{u}-\sum_{u \in c_{2}} G_{u}$ has weight 10 but is not a scalar multiple of the difference of two rows of $G$. There are six words of this type, not counting scalar multiples. Similarly, for $G$ an incidence matrix of $L_{2}(3)$, $C_{p}(G)$ has words of weight 6 that are not scalar multiples of the difference of two rows of $G$. If the bipartite sets of $K_{3,3}$ are $\{1,2,3\},\{4,5,6\}$ then if $s_{1}=\{\{1,4\},\{1,5\},\{2,4\},\{3,6\}\}$, $s_{2}=\{\{1,6\},\{2,5\},\{2,6\},\{3,4\},\{3,5\}\}, w=\sum_{u \in s_{1}} G_{u}-\sum_{u \in s_{2}} G_{u}$ has weight 6 and is not a scalar multiple of the difference of two rows of $G$. There are 36 words of this type, modulo scalar multiples.

Example 34. For the uniform subset graphs $G(n, k, r)$ (see Example 12), since they are edge-transitive, Corollary 30(3), is applicable. In [12] it was shown that $G(n, 1,0)$ (i.e. $K_{n}$ ), which contains triangles for $n \geqslant 3$, satisfies the conclusions of Corollary 30 for $n \geqslant 9$ for $p$ odd, a slight improvement on Corollary 30(3)(i).

Example 35. For $P(9)$ (see Example 13), strongly regular of type $(9,4,1,2), C_{2}(G)$ (where $G$ is an incidence matrix) has words of weight 6 that are not differences of rows of $G$. Similarly $C_{p}(G)$ is covered by Corollary $31(2)$ but not by Corollary $30(2)$, and it has words of weight 6 that are not differences of rows of $G$. These can be obtained from a 6 -cycle $\left(x_{1}, \ldots, x_{6}\right)$ that is an isometric subgraph of $P(9)$, in which case $w=\sum_{i=1}^{6} G_{x_{i}}-\boldsymbol{J}$ has weight 6 and $\operatorname{Supp}(w)=\left\{x_{1} x_{2}, x_{2} x_{3}, x_{3} x_{4}, x_{4} x_{5}, x_{5} x_{6}, x_{6} x_{1}\right\}$. An example of such a cycle is $\left(1, w^{5}, w^{6}, w^{2}, w, w^{4}\right)$ where $w$ is a primitive root of the polynomial $X^{2}+2 X+2$ over $\mathbb{F}_{3}$.

\section{Conclusion}

In this paper we have used the notion of edge-connectivity, and related concepts, and the vast literature devoted to the study of classes of graphs that have the various connectivity properties, to show that for a wide range of classes of $k$-regular connected graphs $\Gamma=$ $(V, E)$, the $p$-ary code, for any prime $p$, generated by the row span over $\mathbb{F}_{p}$ of a $|V| \times|E|$ incidence matrix $G$ for $\Gamma$ has dimension $|V|$ or $|V|-1$ and the properties:

- the minimum weight is $k$ and the vectors of weight $k$ are the scalar multiples of the rows of $G$, i.e. up to scalar multiples, there are $|V|$ words of weight $k$; 
- there are no words of weight $i$ such that $k<i<2 k-2$, and the words of weight $2 k-2$ are the scalar multiples of the differences of two rows of $G$ corresponding to adjacent vertices, i.e. up to scalar multiples, there are $|E|$ words of weight $2 k-2$.

In particular, for graphs satisfying these conditions, non-isomorphic graphs give nonisomorphic codes from their incidence matrices since an incidence matrix of such a graph can be recovered from the words of minimum weight of the code. The work extends the results from [8]. Furthermore, if the graph has an edge-transitive automorphism group, these codes can be used for full error-correction using permutation decoding, as described in $[8$, Result 7].

\section{References}

[1] E. F. Assmus, Jr and J. D. Key. Designs and their Codes. Cambridge: Cambridge University Press, 1992. Cambridge Tracts in Mathematics, Vol. 103 (Second printing with corrections, 1993).

[2] C. Balbuena, A. Carmona, J. Fàbrega, and M. A. Fiol. Superconnectivity of bipartite digraphs and graphs. Discrete Appl. Math., 197/198:61-75, 1999.

[3] C. Balbuena, Y. Lin, and M. Miller. Diameter-sufficient conditions for a graph to be super-resricted connected. Discrete Appl. Math., 156:2827-2834, 2008.

[4] D. Bauer, F. Boesch, C. Suffel, and R. Tindell. Connectivity extremal problems and the design of reliable probabilistic networks. In The Theory and Applications of Graphs, Kalamazoo MI, pages 45-54. Wiley, New York, 1981.

[5] W. Bosma, J. Cannon, and C. Playoust. The Magma algebra system I: The user language. J. Symbolic Comput., 24, 3/4:235-265, 1997.

[6] A. E. Brouwer and W. H. Haemers. Eigenvalues and perfect matchings. Linear Algebra Appl., 395:155-162, 2005.

[7] J. Cannon, A. Steel, and G. White. Linear codes over finite fields. In J. Cannon and W. Bosma, editors, Handbook of Magma Functions, pages 3951-4023. Department of Mathematics, Univ. of Sydney, 2006. V2.13, http://magma.maths.usyd.edu.au/.

[8] P. Dankelmann, J. D. Key, and B. G. Rodrigues. Codes from incidence matrices of graphs. Des. Codes Cryptogr., 68:373-393, 2013. doi:10.1007/s10623-011-9594-x.

[9] P. Erdös, J. Pach, R. Pollack, and Z. Tuza. Radius, diameter and minimum degree. J. Combin. Theory Ser. B, 47(1):73-79, 1989.

[10] J. Fàbrega and M. A. Fiol. Bipartite graphs and digraphs with maximum connectivity. Discrete Appl. Math., 69:271-279, 1996.

[11] M. A. Fiol. On super-edge-connected digraphs and bipartite digraphs. J. Graph Theory, 16:545-555, 1992.

[12] W. Fish, J. D. Key, and E. Mwambene. Codes from uniform subset graphs and line graphs. (In preparation).

[13] W. Fish, J. D. Key, and E. Mwambene. Codes from the incidence matrices and line graphs of Hamming graphs. Discrete Math., 310:1884-1897, 2010. 
[14] D. Ghinelli, M. J. de Resmini, and J. D. Key. Minimum words of codes from affine planes. J. Geom., 91:43-51, 2008.

[15] D. Ghinelli and J. D. Key. Codes from incidence matrices and line graphs of Paley graphs. Adv. Math. Commun., 5:93-108, 2011. doi:10.3934/amc.2011.5.93.

[16] S. L. Hakimi and J. G. Bredeson. Graph theoretic error-correcting codes. IEEE Trans. Inform. Theory, 14:584-591, 1968.

[17] S. L. Hakimi and H. Frank. Cut-set matrices and linear codes. IEEE Trans. Inform. Theory, 11:457-458, 1965.

[18] A. Hellwig and L. Volkmann. Sufficient conditions for graphs to be $\lambda^{\prime}$-optimal, superedge-connected and maximally edge-connected. J. Graph Theory, 48:228-246, 2005.

[19] A.K. Kelmans. Asymptotic formulas for the probability of $k$-connectedness of random graphs. Theory Probab. Appl., 17:243-254, 1972.

[20] J. D. Key, J. Moori, and B. G. Rodrigues. Codes associated with triangular graphs, and permutation decoding. Int. J. Inform. and Coding Theory, 1, No.3:334-349, 2010.

[21] J. D. Key and B. G. Rodrigues. Codes associated with lattice graphs, and permutation decoding. Discrete Appl. Math., 158:1807-1815, 2010.

[22] J. D. Key, W. Fish, and E. Mwambene. Codes from the incidence matrices and line graphs of Hamming graphs $H^{k}(n, 2)$ for $k \geqslant 2$. Adv. Math. Commun., 5:373-394, 2011.

[23] D. Meierling and L. Volkmann. Sufficient conditions for triangle-free graphs to be optimally restricted edge-connected. Discrete Appl. Math., 160:1775-1781, 2012.

[24] J. Ou and F. Zhang. Super-restricted edge-connectivity of regular graphs. Graphs Combin., 21(4):459-467, 2005.

[25] Y. Tian and J. Meng. On super-restricted edge-connectivity of edge-transitive graphs. Discrete Math., 310:2273-2279, 2010.

[26] Y. Tian, J. Meng, and X. Liang. On super-restricted edge-connectivity of half vertex transitive graphs. Graphs Combin., 28:287-296, 2012.

[27] R. Tindell. Edge-connectivity properties of symmetric graphs. Preprint, Stevens Institute of Technology, Hoboken, NJ, 1982.

[28] S. Wang, J. Li, L. Wu, and S. Lin. Neighbourhood conditions for graphs to be super resricted edge connected. Networks, 56(1):11-19, 2010.

[29] Y.Q. Wang. Super-restricted edge-connectivity of vertex-transitive graphs. Discrete Math., 289:199-205, 2004.

[30] W. Yang, Z. Zhang, C. Quin, and X. Guo. On super 2-restricted and 3-restricted edge-connected vertex transitive graphs. Discrete Math., 311:2683-2689, 2011.

[31] J. Yuan, A. Liu, and S. Wang. Sufficient condtitions for bipartite graphs to be super-k-restricted edge connected. Discrete Math., 309:2886-2896, 2009.

[32] J. X. Zhou. Super-restricted edge-connectivity of regular edge-transitive graphs. Discrete Appl. Math., 160:1248-1252, 2012. doi:10.1016/j.dam.2011.12.004. 